\title{
Super-resolution with Nonlocal Regularized Sparse Representation
}

\author{
Weisheng Dong ${ }^{\mathrm{a}}$, Guangming $\mathrm{Shi}^{\mathrm{a}}$, Lei Zhang ${ }^{\mathrm{b}}$, and Xiaolin $\mathrm{Wu}^{\mathrm{c}}$ \\ ${ }^{a}$ Key Laboratory of Intelligent Perception and Image Understanding (Chinese Ministry of Education), School \\ of Electronic Engineering, Xidian University, China \\ ${ }^{b}$ Dept. of Computing, The Hong Kong Polytechnic University, Hong Kong \\ ${ }^{c}$ Dept. of Electrical and Computer Engineering, McMaster University, Canada
}

\begin{abstract}
The reconstruction of a high resolution (HR) image from its low resolution (LR) counterpart is a challenging problem. The recently developed sparse representation (SR) techniques provide new solutions to this inverse problem by introducing the $l_{1}$-norm sparsity prior into the super-resolution reconstruction process. In this paper, we present a new SR based image super-resolution by optimizing the objective function under an adaptive sparse domain and with the nonlocal regularization of the HR images. The adaptive sparse domain is estimated by applying principal component analysis to the grouped nonlocal similar image patches. The proposed objective function with nonlocal regularization can be efficiently solved by an iterative shrinkage algorithm. The experiments on natural images show that the proposed method can reconstruct HR images with sharp edges from degraded LR images.
\end{abstract}

Keywords: sparse representation, nonlocal self-similarity, super-resolution, iterative shrinkage algorithm

\section{INTRODUCTION}

Image super-resolution aims to reconstruct a high resolution (HR) image from its degraded and down-sampled low resolution (LR) counterparts. It has wide applications in computer vision, image enhancement, medical imaging and high definition televisions. A typical image super-resolution process consists of LR image denoising, interpolation and deblurring, subjecting to a known imaging model of the LR image. The LR imaging process can be modeled by:

$$
\mathbf{y}=D H \mathbf{x}+\mathbf{n}
$$

where $H$ is a blurring operator that mimics the point spread function of a camera, $D$ is a down-sampling operator, $\mathbf{n}$ is the noise introduced in the LR image generation, $\mathbf{x}$ is the target HR image, and $\mathbf{y}$ is the observed LR image. The reconstruction of the original HR image is a typical ill-posed inverse problem. This problem becomes even more underdetermined in the case that only one LR image is available. In this paper, we concentrate on the image superresolution from only a single image.

Conventional linear interpolation based methods, such as bilinear, bicubic and cubic spline interpolators, reconstruct HR images with jaggy and zipping artifacts. To improve the linear interpolators, directional interpolators [1]-[3] have been proposed to perform the interpolation along the edge directions. Especially, in [3] Zhang and Wu optimize the interpolator based on the local covariance of the image signal and achieve state-of-the-art interpolation results. However, all these interpolation based approaches do not handle the blurring and noises in the LR images. Additional steps have to be carried out to remove the blurring and noises in the LR images. However, the separated denoising and deblurring steps do not sufficiently exploit the information hidden in the imaging model in Eq. (1).

Another classical super-resolution approach is iterative back-projection (IBP) [4], which is designed based on the imaging model in Eq. (1). However, the IBP process involves much uncertainty in recovering the HR images, and hence chessboard and zipping artifacts are commonly observed in the reconstructed HR images by IBP algorithm. This mainly because IBP tries to find the $\mathbf{x}$ that could minimize $\|\mathbf{y}-D H \mathbf{x}\|_{2}$; however, there could be many possible candidates of $\mathbf{x}$ that could make $\|\mathbf{y}-\mathrm{DH} \mathbf{x}\|_{2}$ very small. In other words, the solution space of IBP is too big so that the resulting solution may not be the best one.

To improve the performance of the IBP method, image prior knowledge that take into account the local image edge geometries and nonlocal image redundancies has been incorporated into the IBP process in [5] [6]. The regularized IBP techniques by bilateral filters [5] and nonlocal means based filters [6] can remove many artifacts of the HR images generated by the original IBP method. However, their performances are still not very satisfying in recovering fine image 
details and suppressing noises. To reconstruct more visually pleasing HR images, more prior knowledge of natural images should be used to reduce the uncertainty of the HR images. Typically, the regularized super-resolution can be formulated by solving the following minimization problem:

$$
\underset{\mathbf{x}}{\arg \min }\|\mathbf{y}-D H \mathbf{x}\|_{2}+\lambda J(\mathbf{x})
$$

where $J(\mathbf{x})$ is a regularization term specifying the prior knowledge of the HR image and $\lambda$ is a scalar balancing between the quadratic fidelity term and the regularization term. A well-known regularization prior is the minimum pixel intensity total variations (TV) [7]. The TV-regularized approaches favor the images with piecewise smooth edge structures in the solution space of $\mathbf{x}$; however, the TV-based super-resolution techniques cannot recover image fine details and often have staircase artifacts. Other image priors, such as edge smoothness [8] and gradient profile priors [9] have been proposed for image super-resolution, yet the resulting edges look unnatural.

A recently proposed image prior knowledge is sparsity of natural images. It assumes that a natural image can be sparsely represented in some specific domain (e.g. wavelet domain, Fourier domain), or it has a sparse expansion over a dictionary of atoms, i.e. $\mathbf{x}=\Psi \alpha$ and most of the coefficients in $\alpha$ are nearly zero. In general, the sparsity constrain on $\alpha$ is implemented by requiring that the $l_{1}$-norm of $\boldsymbol{\alpha}$ is small enough, i.e. $\|\boldsymbol{\alpha}\|_{1}<t$. The sparse representation (SR) techniques have been successfully applied to a series of inverse problems, including image deblurring [10], denoising [11] and compressive sensing [12]. In [13], the SR of the HR image over a learned dictionary was proposed to regularize the image super-resolution process. As a learning-based method, its performance relies on the training set, and the learning of a universal dictionary for SR is very complicated.

In this paper, we present a new SR based model to reconstruct an HR image from its LR counterpart. The contributions of the proposed approach are twofold. First, we propose to adaptively estimate the sparse domain of the HR image patches using adaptive principal component analysis (PCA) via non-local similar patch grouping. Second, to further enhance the performance of the proposed approach, a nonlocal self-similarity quadratic constrain is also introduced to fully exploit the nonlocal image redundancies. Since both the two points use the non-local information of the image, we call the proposed method non-local regularized SR for image super-resolution. In addition, the proposed minimization problem with non-local regularizations can be efficiently solved by using a new family of numerical algorithms, called iterative shrinkage algorithms [14] [15].

The rest of the paper is organized as follows. Section 2 presents the proposed algorithm in detail. Section 3 conducts experiments to validate the performance of the proposed method. Section 4 concludes the paper.

\section{THE PROPOSED IMAGE SUPER RESOLUTION APPROACH}

The sparsity prior of natural images in a specific transform domain is an effective constrain to refine the solution space of image super-resolution. Wavelet transform, DCT, curvelet and contourlet transforms are commonly used transforms for sparse image representation. However, these transforms use a fixed set of bases, which lack flexibilities in adapting to various complex local structures in natural images. Therefore, dictionary learning [16] techniques have been proposed to learn a universal over-complete dictionary of atoms so that the image can be sparsely coded via $l_{1}$-norm minimization. However, for each image local patch, there are too many irrelative atoms in the universal dictionary, which degrades the effectiveness and efficiency of sparse representation (SR) and hence degrades the image reconstruction performance. It is of high demand that we could adaptively determine the sparse domain of each local patch.

In [17] [18], the principal component analysis (PCA) technique was used to adaptively de-correlate the image local structures for noise removal. For each image block, a PCA transformation matrix is locally computed. To this end, a set of image blocks that are similar to the current image block is grouped and a PCA transform matrix is computed over the training dataset. The PCA transformation matrix actually defines a type of image local sparse domain because the image local patch can be well reconstructed by using only a few significant principal components. Different from the wavelet transform, DCT, etc, such a PCA transform is signal adaptive. In [16-17], state-of-the-art image denoising results have been obtained by thresholding in the adaptive PCA domains. Inspired by the work in [16-17], in this paper we propose to use the locally adaptive PCA transform as the adaptive sparse domain. On the other hand, the nonlocal self-similarity prior is used as regularization term in the SR based image reconstruction. Finally, an objective function will be 
constructed, which is an $l_{1}$-norm and $l_{2}$-norm compounded SR minimization problem. Fortunately, an efficient numerical algorithm can be readily obtained by using the recently developed iterative shrinkage (IS) techniques [14] [15].

\subsection{Adaptive sparse domain determination by local PCA transform}

To adaptively compute the sparse domain of each local patch, we model a local image patch as a vector variable and then calculate its statistics by using its available samples. Specifically, the adaptive PCA transformation method proposed in [17] [18] is used here to determine the adaptive sparse domain of each local patch. Denote by $\vec{x}=\left[x_{1}, x_{2}, \cdots, x_{m}\right]^{T}$ a vector variable containing all pixel values within a $w \times w$ image patch. To compute the PCA transformation matrix of $\vec{x}$, a training dataset of it is needed. Denote by

$$
X=\left[\begin{array}{cccc}
x_{1,0} & x_{1,1} & \cdots & x_{1, n-1} \\
x_{2,0} & x_{2,1} & \cdots & x_{2, n-1} \\
\vdots & \vdots & \vdots & \vdots \\
x_{m, 0} & x_{m, 1} & \cdots & x_{m, n-1}
\end{array}\right]
$$

a sample matrix of variable $\vec{x}$, where the $i^{\text {th }}$ row of sample matrix $X$, denoted by $X_{i}=\left[\begin{array}{llll}x_{i, 0} & x_{i, 1} & \cdots & x_{i, n-1}\end{array}\right]$, is the sample vector of variable $x_{i}$. The sample vector $X_{i}$ is centralized as $\bar{X}_{i}=X_{i}-\mu_{i}$, where $\mu_{i}$ is the mean of $X_{i}$. Other rows of $X$ can be centralized analogously and we denote by $\bar{X}$ the centralized matrix of $X$. With $\bar{X}$, the covariance matrix of $X$ is calculated by

$$
\Omega=\frac{1}{n} \bar{X} \bar{X}^{T}
$$

The goal of PCA is to find an orthonormal transformation matrix $P$ to decorrelate $\bar{X}, \bar{Y}=P \bar{X}$, so that the covariance matrix of $\bar{Y}$ is diagonal. Since $\Omega$ is symmetrical, by apply the SVD (singular value decomposition) to it, we have

$$
\Omega=\Phi \Lambda \Phi^{T}
$$

where $\Phi=\left[\begin{array}{llll}\phi_{1} & \phi_{2} & \cdots & \phi_{m}\end{array}\right]$ is the $m \times m$ orthonormal eigenvector matrix and $\Lambda=\operatorname{diag}\left\{\lambda_{1}, \lambda_{2}, \ldots, \lambda_{m}\right\}$ is the diagonal eigenvalue matrix with $\lambda_{1} \geq \lambda_{2} \geq \ldots \geq \lambda_{m}$. The terms $\phi_{1}, \phi_{2}, \ldots, \phi_{m}$ and $\lambda_{1}, \lambda_{2}, \ldots, \lambda_{m}$ are the eigenvectors and eigenvalues of $\Omega$. By setting $P=\Phi^{T}, \bar{X}$ can be decorrelated, i.e. $\bar{Y}=P \bar{X}$ and $\Lambda=\frac{1}{n} \overline{Y Y}^{T}$. An important property of PCA is that it fully de-correlates the original dataset $\bar{X}$. Most of the energy of a signal will concentrate on a small subset of the PCA transformed dataset.

To apply the block-based adaptive PCA transform for the given image, we need a set of training sample for each image patch $\vec{x}$ so that the PCA transformation matrix $P$ can be computed. To this end, we can collect every possible image patches in a large window centered on $\vec{x}$. However, there can be many different image patches from the current one in the search area, which will deteriorate the estimation of the covariance and hence the PCA matrix. To exclude these irrelative training samples, a block matching method is used to select the image patches with similar spatial structures. The image patches whose Euclid distances to $\vec{x}$ are smaller than a predefined threshold are selected: $\left\|\vec{x}_{i}-\vec{x}\right\|_{2}<\tau$. By such constraint, nonlocal image information can be used to compute the PCA transformation matrixes.

The ordering property of PCA bases allows a good reconstruction of the image by using only a few principal components, which naturally provide an adaptive sparse domain of each local patch. By allowing the overlapping of the image patches, pixels in overlapped region will be transformed into different PCA domains. This forms a redundant representation of image signals, which is very helpful in suppressing noises.

\subsection{Sparse representation with adaptive sparse domain}

Since the problem of image super-resolution involves severe uncertainties, prior knowledge is required to regularize the solution. The proposed SR based image super-resolution using adaptive PCA transformations can be formulated by

$$
\underset{\mathbf{x}}{\arg \min }\|\mathbf{y}-D H \mathbf{x}\|_{2}+\lambda \sum_{i}\left\|P_{i} \vec{x}_{i}\right\|_{1}
$$


where $\vec{x}_{i}$ is the vector of the $i^{\text {th }}$ image patch and $P_{i}$ is the computed PCA transformation matrix of $\vec{x}_{i} \cdot \lambda$ is a scalar that balances the $l_{1}$-norm and $l_{2}$-norm terms. A problem of (6) is that the computation of $P_{i}$ requires that the image signal $\mathbf{x}$ should be estimated first. However, the estimation of $\mathbf{x}$ with Eq. (6) requires that $P_{i}$ is available. This is a chicken and egg dilemma. To solve this problem, we initially reconstruct $\mathbf{x}$ by setting $P_{i}$ as a wavelet transformation matrix. Once $\mathbf{x}$ is initialized, $P_{i}$ can be estimated, and subsequently $\mathbf{x}$ can be updated by solving Eq. (6). With the updated $\mathbf{x}$, the PCA transformation matrixes $P_{i}$ can be further updated. These procedures can be iterated to alternatively optimize the reconstructed HR image and the transformation matrixes.

\subsection{Regularization with non-local self-similarity constraint}

The adaptive block-based PCA transformation can better characterize the various local image structures and hence an adaptive sparse representation can be achieved, which is very helpful to improving the image reconstruction performance. However, the estimated PCA transformation matrixes may not be very accurate due to the limited information of the original HR image. This will deteriorate the performance of the proposed approach. To improve the quality of the reconstructed HR images, more prior information should be incorporated.

One important image prior is that natural images often contain repetitive patterns and structures throughout the image. Such non-local statistical redundancies can be very useful in enhancing the quality of reconstructed images. Actually, in the PCA-based adaptive sparse domain determination process, the non-local redundancy information has been already used for training dataset construction. Inspired by the success of the non-local means filtering for image denoising [19], we further introduce a nonlocal self-similarity quadratic constraint into the super-resolution process to fully exploit the nonlocal redundancies. With this quadratic constraint as another regularization term, the image superresolution model in Eq. (6) is lifted to:

$$
\underset{\mathbf{x}}{\arg \min }\|\mathbf{y}-D H \mathbf{x}\|_{2}+\eta \sum_{i}\left\|\vec{x}_{i}-\sum_{j} \gamma_{j}^{i} \vec{x}_{j}^{i}\right\|_{2}+\lambda \sum_{i}\left\|P_{i} \vec{x}_{i}\right\|_{1}
$$

where $\eta$ is a scalar parameter to balance the non-local regularization term; $\vec{x}_{j}^{i}$ is the $j^{\text {th }}$ similar (vectorized) block to $\vec{x}_{i}$ in a nonlocal neighborhood; $\gamma_{j}^{i}$ is the weight assigned to $\vec{x}_{j}^{i}$. We use the block matching method to locate the similar

blocks to $\vec{x}_{i}$ in a large enough window: $\left\|\vec{x}_{i}-\vec{x}_{j}^{i}\right\|_{2}<T$, where $T$ is a predefined threshold. The weights $\boldsymbol{\gamma}_{j}^{i}$ depend on the similarity between $\vec{x}_{i}$ and $\vec{x}_{j}^{i}$, which can be calculated by:

$$
\gamma_{j}^{i}=\frac{1}{c_{i}} \cdot e^{-\frac{\left\|\bar{x}_{i}-x_{j}^{i}\right\|_{2}}{h}}
$$

where $h$ is a scalar controlling the similarity and $c_{i}$ is the normalization factor. As in section 2.2, in Eq. (7) the selection of similar nonlocal neighbors and the computation of the weights require that an initial estimate of the original HR image, and then Eq. (7) can be iteratively solved.

\subsection{Numerical algorithm}

Eq. (7) is a minimization problem with compound regularization terms. For the convenience of expression, we can rewrite Eq. (7) in a matrix form as follows

$$
\underset{\mathbf{x}}{\arg \min }\|\mathbf{y}-D H \mathbf{x}\|_{2}+\eta\|(I-A) \mathbf{x}\|_{2}+\lambda\|P \mathbf{x}\|_{1}
$$

where $P$ is form by all the $P_{\mathrm{i}} ; A$ is a matrix of dimension of $N \times N$ with $N$ being the dimension of the target HR image. $A$ is set as follows

$$
A(m, n)=\left\{\begin{array}{l}
\gamma_{j}^{i}, \text { if } \vec{x}_{n}^{m} \text { is the } j^{t h} \text { similar neighbor to } \vec{x}_{m}, i \text { and } j \text { are the locations of } \vec{x}_{m} \text { and } \vec{x}_{n}^{m} \\
0, \text { otherwise }
\end{array}\right.
$$

Eq. (9) can be expressed as 
Let

$$
\arg \min _{\mathbf{x}}\left\|\left[\begin{array}{l}
\mathbf{y} \\
\mathbf{0}
\end{array}\right]-\left[\begin{array}{l}
D H \\
\eta(I-A)
\end{array}\right] \mathbf{x}\right\|_{2}+\lambda\|P \mathbf{x}\|_{1}
$$

$$
\tilde{\mathbf{y}}=\left[\begin{array}{l}
\mathbf{y} \\
\mathbf{0}
\end{array}\right], K=\left[\begin{array}{l}
D H \\
\eta(I-A)
\end{array}\right], \mathbf{u}=P \mathbf{x}
$$

Since $P$ is orthogonal, we have $\mathbf{x}=P^{T} \mathbf{u}$. Then (11) can be rewritten as follows

$$
\arg \min _{\mathbf{u}}\left\|\tilde{\mathbf{y}}-K P^{T} \mathbf{u}\right\|_{2}+\lambda\|\mathbf{u}\|_{1}
$$

Eq. (13) is a challenging large-scale compound $l_{1}$-norm and $l_{2}$-norm minimization problem. Conventional optimization techniques, such as steepest-descent, conjugate gradient and interior-point algorithms, are inefficient in solving this minimization problem. In this paper, we adopt the recently proposed iterative shrinkage algorithm [14] [15] to solve this minimization problem. We summarize the iterative algorithm for solving (13) in Algorithm 1.

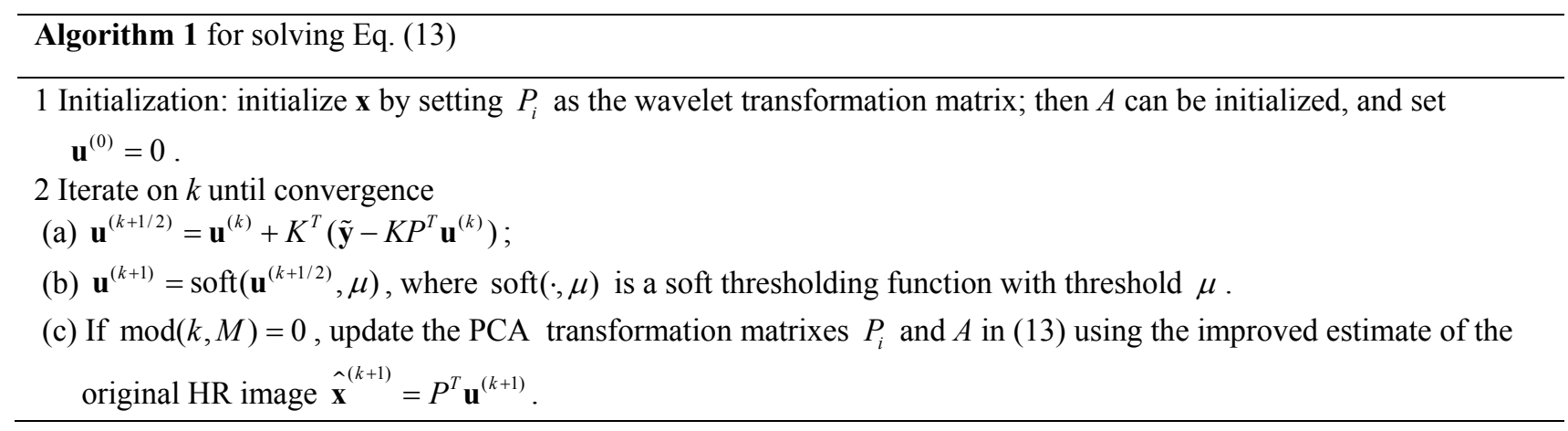

In Algorithm 1, we update $P_{i}$ and $A$ in every $M$ iterations to reduce the computational complexity.

\section{EXPERIMENTAL RESULTS}

In this section, we conduct experiments to verify the efficiency of the proposed technique for image super resolution. The degraded LR images are generated by first applying a blur kernel and then down-sampling. The blurring kernel in the simulations is a $7 \times 7$ Gaussian filter with standard deviation of 1.6. We magnify the LR images by a factor of 3 , which is common in the literature of super-resolution. We use $5 \times 5$ HR image patches with overlap of 1 pixel between adjacent patches when transforming HR images into adaptive PCA domains. These $5 \times 5$ patches are also used to locate the nonlocal similar neighbors. For color images, the proposed approach is only applied to the luminance component and bicubic interpolator is used for the chromatic components.

We compare the proposed approach with some state-of-the-art image super-resolution approaches, including the iterative back-projection (IBP) [4], the softcuts based method in [8], and the SR based method in [13] ${ }^{1}$. The visual results by these competing approaches are presented through Fig .1 Fig. 3. From these figures we can see that the IBP method reconstructs the HR images with jaggy and chessboard artifacts. The HR images reconstructed by the SoftCuts based method remove most of such artifacts but they are over-smoothed and many image details are eliminated. The approach in [13] is competitive in visual quality. However, the reconstructed edges and textures by it are not smooth. Chessboard artifacts and noises can be observed in the edge regions. The reason is that it heavily relies on the training data and tends to generate inconsistencies between adjacent image patches. Without any exceptions, the proposed approach reconstructs the most visually pleasant HR images. The edges and textures reconstructed by our approach are much sharper and cleaner than others. Also, more image details are recovered by our approach.

${ }^{1}$ We thank the authors of [8] and [13] for providing their code or experimental results. 
In practical LR imaging process, noise is often introduced. To demonstrate the robustness of the proposed method to noise, we add the Gaussian noises with standard deviation of 5 to the simulated LR images. The HR images produced by the competing approaches are shown in Fig. 4 and Fig. 5. We can see that the IBP method magnify the noise since the back-projection process is very sensitive to noise. The noise is mostly removed by the Softcuts and the SR-based methods in [8] and [13]. However, the image details are also removed in their results. The proposed approach can well handle the super-resolution and denoising simultaneously. As shown in Fig. 4 and Fig. 5, not only the noises are well suppressed, but also sharp edges and textures are well preserved. The PSNR results of the reconstructed images in Fig. 1 Fig. 5 are shown in Table 1, from which we can see that the PSNR values by the proposed method are much higher that others.

\section{CONCLUSION}

This paper presented a sparse representation (SR) based image super-resolution approach by maximizing the sparsity of the HR images in adaptive sparse domains. The sparse domain of an image patch was locally determined by applying principle component analysis (PCA) of the nonlocal similar image patches. The sparsity of the HR images was enforced by an $l_{1}$-norm regularization term that penalizes the principle components of the image signals. In addition, a nonlocal similarity prior of natural images was also incorporated to exploit the nonlocal image redundancies. The proposed objective function with compound $l_{1}$-norm and $l_{2}$-norm regularization terms can be efficiently solved by an iterative shrinkage algorithm. Experimental results demonstrated that the proposed approach can reconstruct sharp edges and fine image details and is robust to noises.

\section{ACKNOWLEDGEMENTS}

This work is supported by the Program for New Century Excellent Talents (No. NCET-07-0656), the NSF China (No. 60736043, 60776795, 60805012 and 60902031), and the Ph.D. Program Foundation of Ministry of Education of China (Nos. 200807010004, 20090203110003).

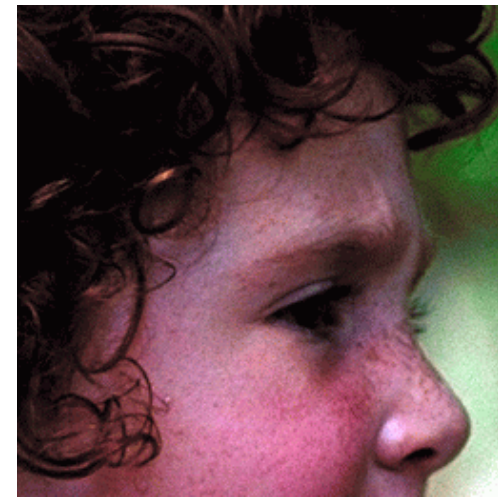

(a) Original

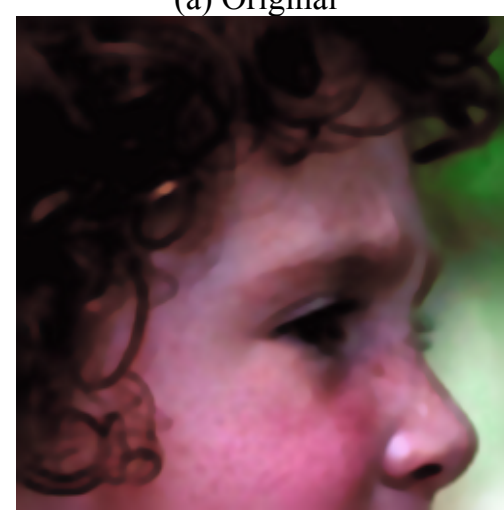

(d) SoftCuts [8]

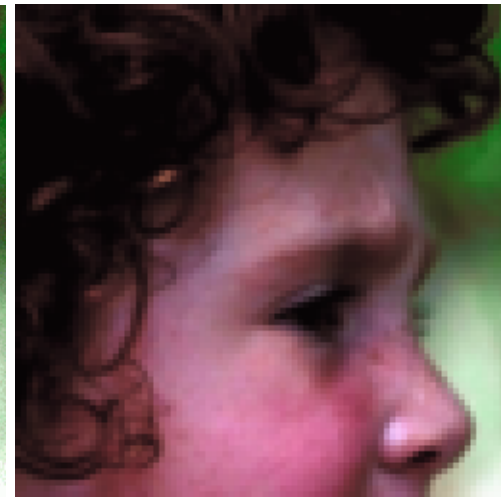

(b) Input LR image

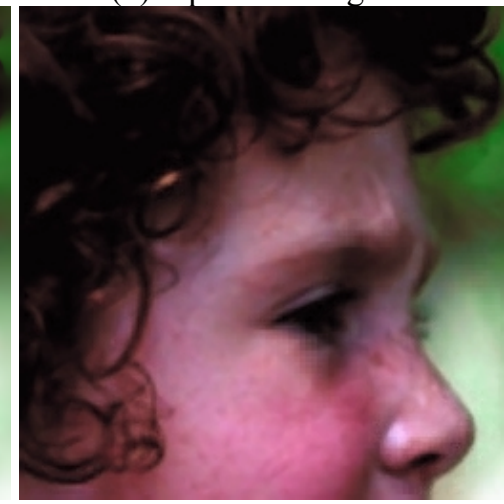

(e) Sparse representation [13]

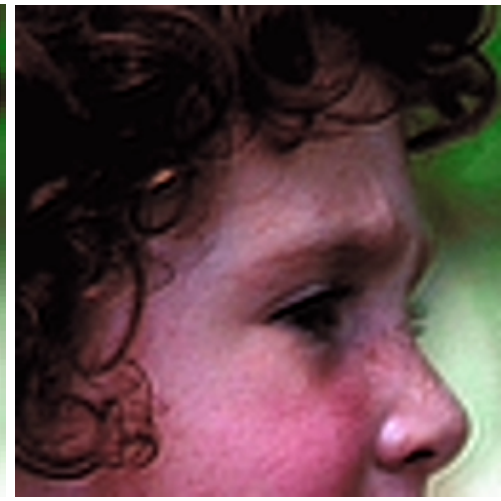

(c) Back-projection [4]

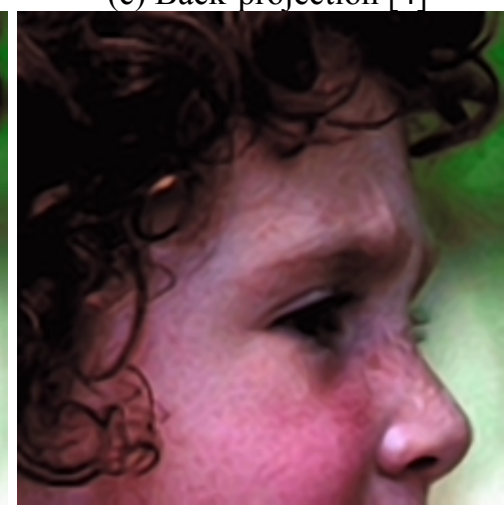

(f) Proposed

Fig. 1 Results on the Girl image with scaling factor 3. 


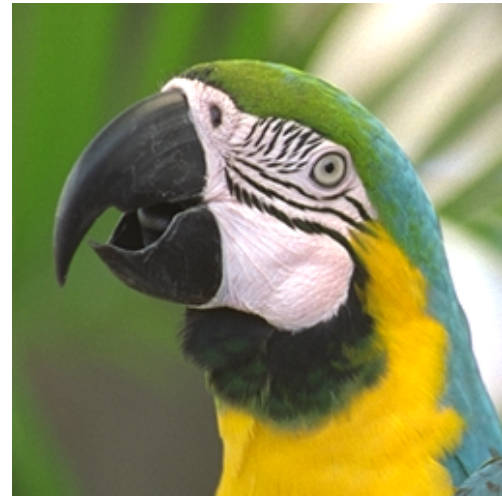

(a) Original

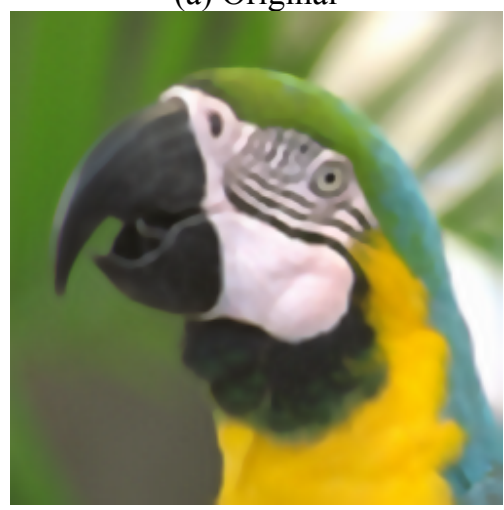

(d) SoftCuts [8]

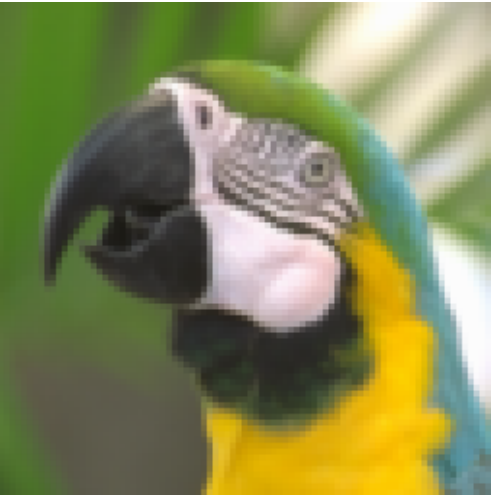

(b) Nearest neighbor

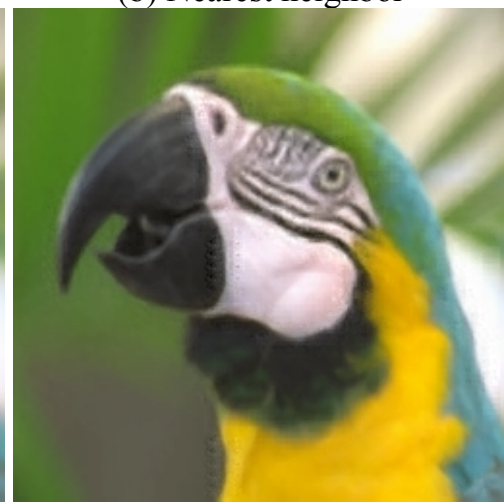

(e) Sparse representation [13]

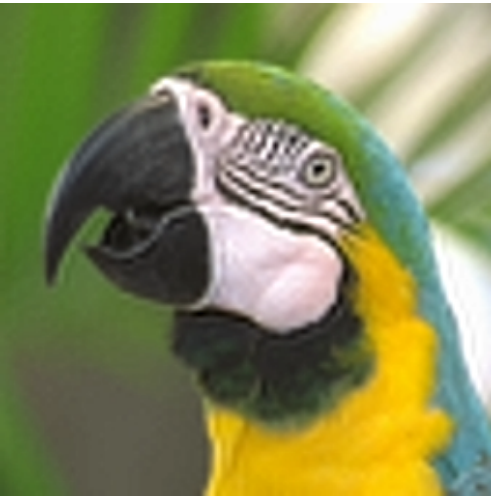

(c) Back-projection [4]

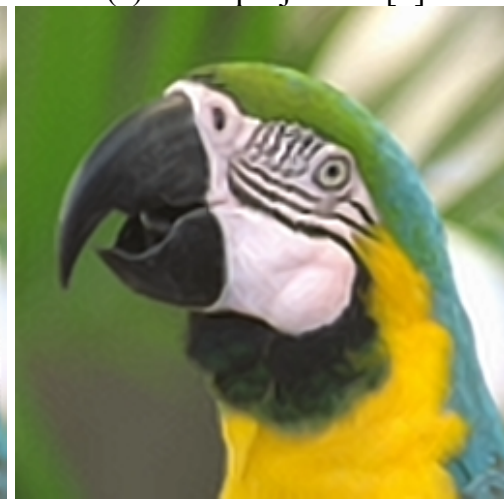

(f) Proposed

Fig. 2 Results on the Parrot image with scaling factor 3.

Table 1 The PSNR (dB) results of the luminance components reconstructed by different methods.

\begin{tabular}{|c|c|c|c|c|}
\hline Images & IBP [4] & SoftCuts [8] & {$[13]$} & Proposed \\
\hline Girl & 31.8 & 32.0 & 31.9 & $\mathbf{3 3 . 3}$ \\
\hline Parrot & 27.2 & 27.7 & 27.7 & $\mathbf{2 9 . 6}$ \\
\hline Butterfly & 23.7 & 25.2 & 23.8 & $\mathbf{2 6 . 9}$ \\
\hline Noisy Parthenon & 24.9 & 25.6 & 25.4 & $\mathbf{2 5 . 9}$ \\
\hline Nosy Parrot & 26.4 & 27.4 & 27.4 & $\mathbf{2 8 . 1}$ \\
\hline
\end{tabular}




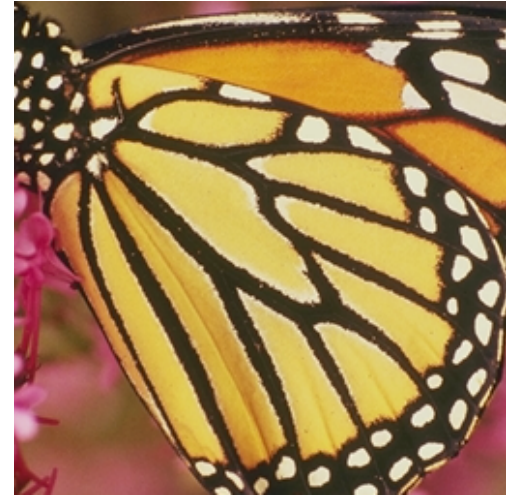

(a) Original

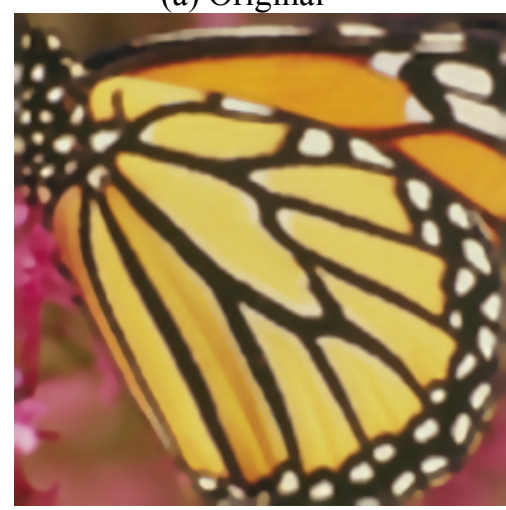

(d) SoftCuts [8]

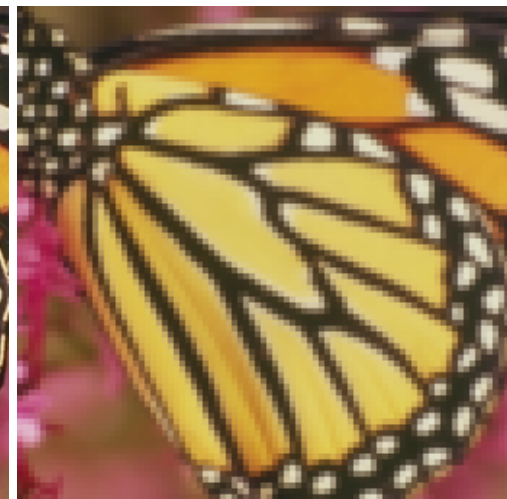

(b) Input LR image

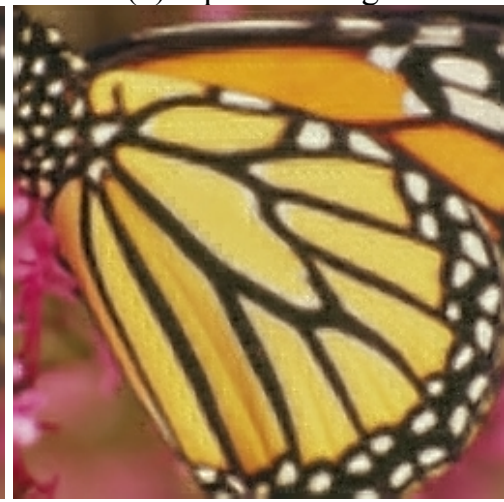

(e) Sparse representation [13]

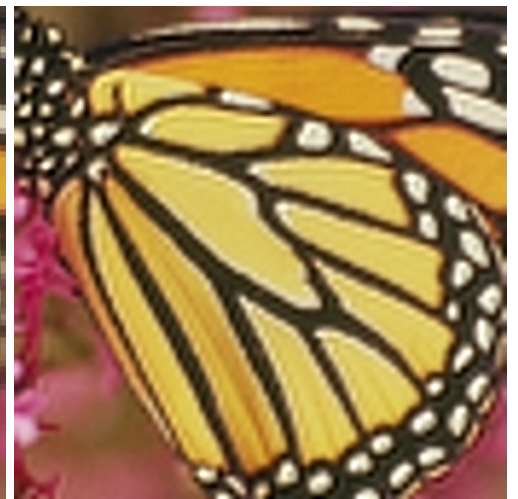

(c) Back-projection [4]

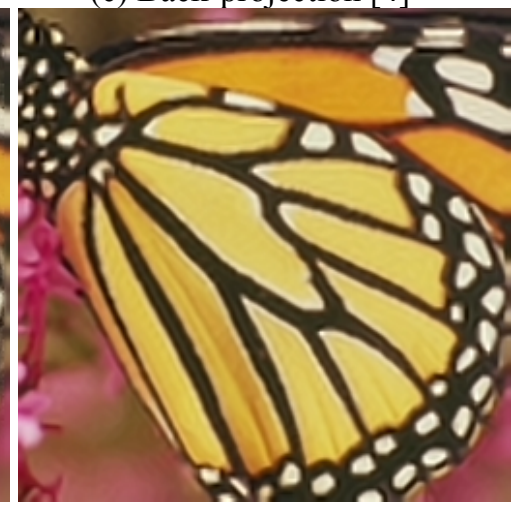

(f) Proposed

Fig. 3 Results on the Butterfly image with scaling factor 3.

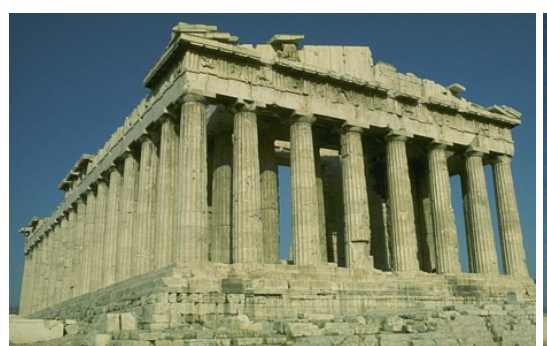

(a) Original

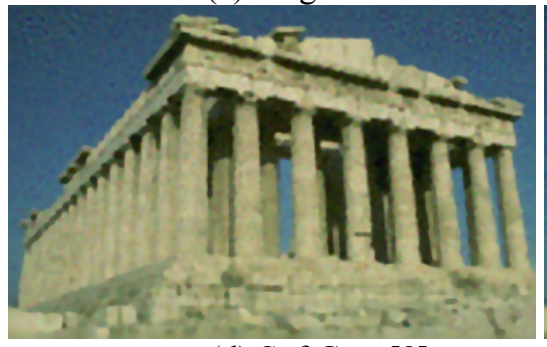

(d) SoftCuts [8]

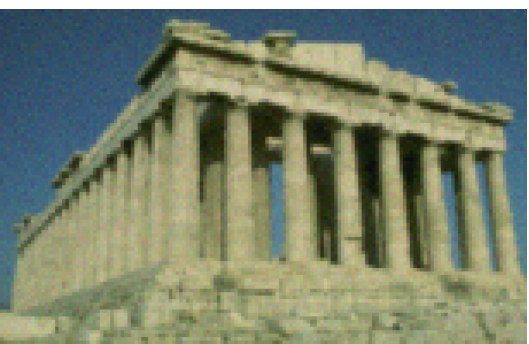

(b) Input LR image

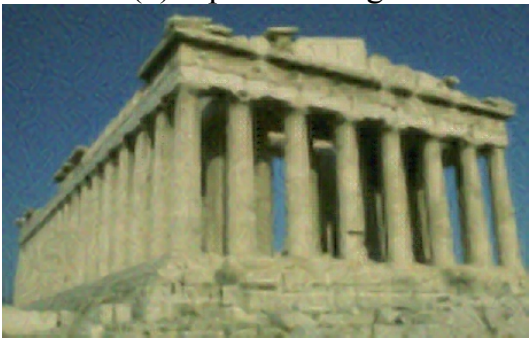

(e) Sparse representation [13]

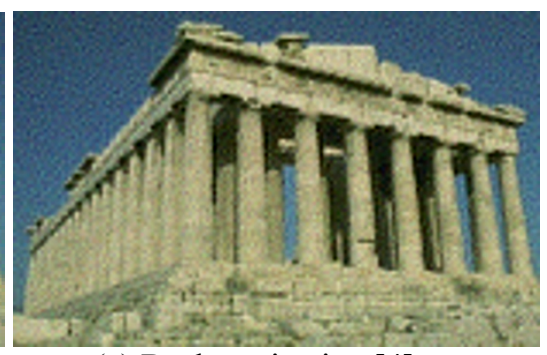

(c) Back-projection [4]

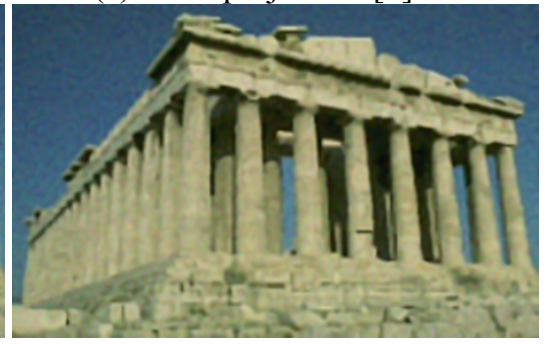

(f) Proposed

Fig. 4 Results on the noisy Parthenon image with scaling factor 3. The standard deviation of Gaussian noise is 5. 


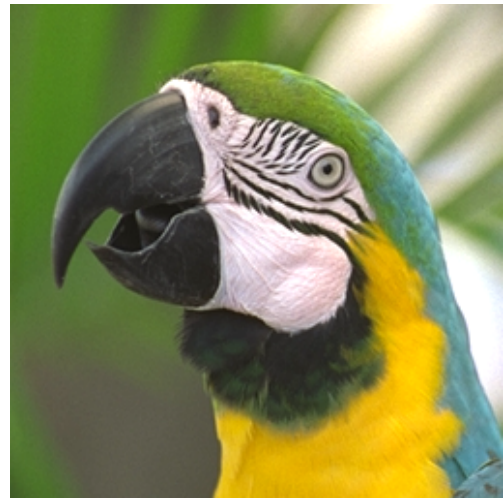

(a) Original

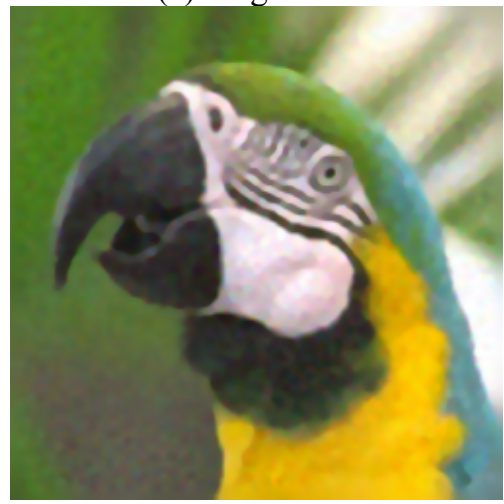

(d) SoftCuts [8]

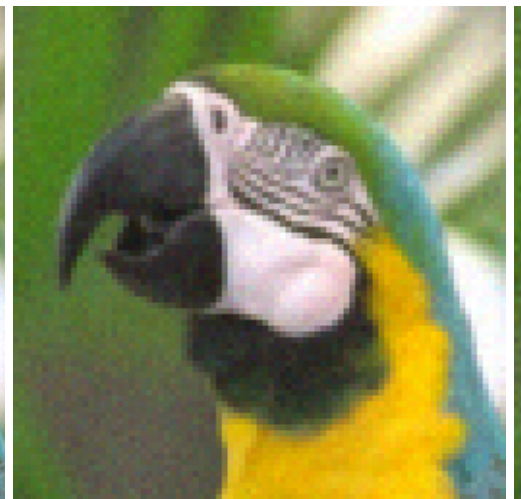

(b) Input LR image

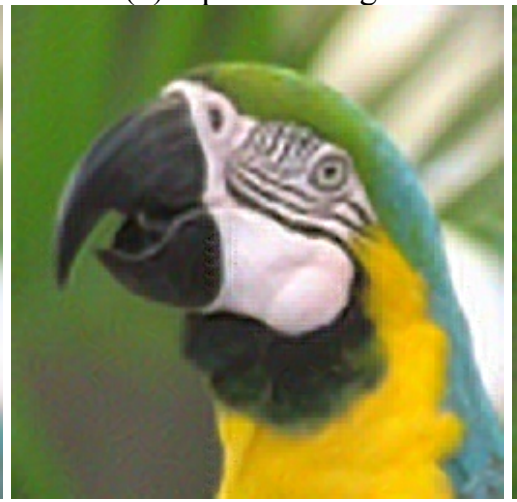

(e) Sparse representation [13]

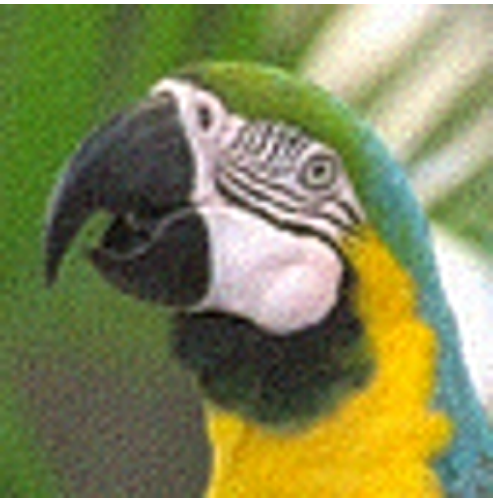

(c) Back-projection [4]

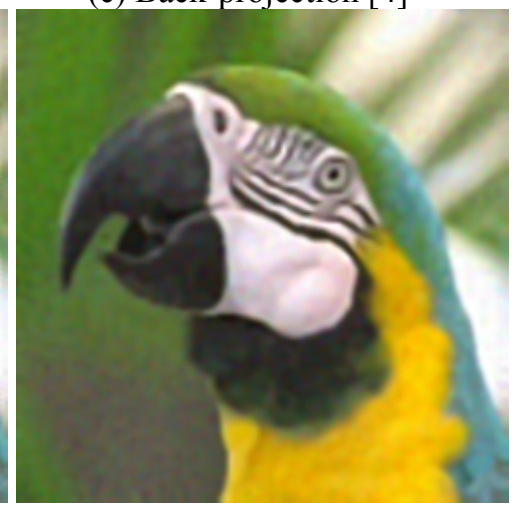

(f) Proposed

Fig. 5 Results on the noisy Parrot image with scaling factor 3. The standard deviation of Gaussian noise is 5.

\section{REFERENCES}

1. X. Li and M. T. Orchard, "New edge-directed interpolation" IEEE Trans. Image Process., vol. 10, no. 10, pp. 15211527, Oct. 2001.

2. L. Zhang and $\mathrm{X}$. Wu, "An edge-guided image interpolation algorithm via directional filtering and data fusion," IEEE Trans. Image Process., vol. 15, no. 8, pp. 2226-2238, Aug. 2006.

3. X. Zhang and $\mathrm{X}$. Wu, "Image interpolation by adaptive 2D autoregressive modeling and soft-decision estimation," IEEE Trans. Image Process., vol. 17, no. 6, pp. 887-896, Jun. 2008.

4. M. Irani and S. Peleg, "Motion analysis for image enhancement: resolution, occlusion and transparency," JVCI., 1993.

5. S. Dai, M. Han, Y. Wu, and Y. Gong, "Bilateral back-projection for single image super resoltuion" in Proc. Int. Conf. on Multimedia and Expo, 2007, pp. 1039-1042, July 2007.

6. W. Dong, L. Zhang, G. Shi, and X. Wu, "Nonlocal back-projection for adaptive image enlargement", in Proc. Int. Conf. Image Process.,2009, Oct. 2009.

7. S. Farsiu, M. D. Robinson, M. Elad, and P. Milanfar, "Fast and robust multiframe super-resolution," IEEE Trans. Image Process., vol. 15, no. 1, pp. 141-159, Jan. 2006.

8. S. Dai, M. Han, W. Xu, Y. Wu, Y. Gong, and A. K. Katsaggelos, "SoftCuts: a soft edge smoothness prior for color image super-resolution," IEEE Trans. Image Process., vol. 18, no. 5, pp. 969-981, May 2009.

9. J. Sun, J. Sun, Z. Xu, and H. Shum, "Image super-resolution using gradient profile prior," in Proc. IEEE Computer Vision and Pattern Recognition, pp. 1-8, Jun. 2008.

10. J. Cai, H. J, C. Liu and Z. Shen, "Blind motion deblurring from a single image using sparse approximation," in Proc. IEEE Computer Vision and Pattern Recognition, Jun. 2009. 
11. M. Elad and M. Aharon, "Image denoising via sparse and redundant representations over learned dictionaries," IEEE Trans. Image Process., vol. 15, no. 12, pp. 3736-3745, Dec. 2006.

12. E. J. Candes and M. B. Wakin, "An introduction to compressive sampling," IEEE Signal Process. Mag., vol. 25, no. 2, pp. 21-30, Mar. 2008.

13. J. Yang, J. Wright, Y. Ma, and T. Huang, "Image super-resolution as sparse representation of raw image patches," IEEE Computer Vision and Pattern Recognition, vol. 1, pp. 1-8, Jun. 2008.

14. I. Daubechies, M. Defrise, and C. De. Mol, "an iterative thresholding algorithm for linear inverse problems with sparsity constraint," Coтmu. On Pure and Applied Math., vol. 57, no. 11, pp. 1413-1457, Aug. 2004.

15. P. Combettes, and V. Wajs, "Signal recovery by proximal forward-backward splitting," SIAM J. Multiscale Model Simul., vol. 4, pp. 1168-1200, 2005.

16. M. Aharon, M. Elad, and A. Bruckstein, "K-SVD: an algorithm for designing overcompleter dictionaries for sparse representation," IEEE Trans. Signal Process., vol. 54, no. 11, pp. 4311-4322, Nov. 2006.

17. L. Zhang, R. Lukac, X. Wu, and D. Zhang, "PCA-based spatially adaptive denoising of CFA images for singlesensor digital cameras," IEEE Trans. Image Process., vol. 18, no. 4, pp. 797-812, Apr. 2009.

18. L. Zhang, W. Dong, D. Zhang, and G. Shi, "Two-stage image denoising by principal component analysis with local pixel grouping," to appear in Pattern Recognition.

19. A. Buades, B. Coll, and J. M. Morel, "A review of image denoising algorithms, with a new one," Multisc. Model. Simulat., vol. 4, no. 2, pp. 490.530, 2005. 